\title{
The canine epiphyseal-derived mesenchymal stem cells are comparable to bone marrow derived-mesenchymal stem cells
}

\author{
Ya-Pei CHANG ${ }^{1)}$, Hsuan-Ping HONG ${ }^{1)}$, Yen-Hua LEE ${ }^{2)}$ and I-Hsuan LIU ${ }^{2,3) *}$ \\ 1) Institute of Veterinary Clinical Science, School of Veterinary Medicine, National Taiwan University, Taipei 106, Taiwan \\ ${ }^{2)}$ Department of Animal Science and Technology, National Taiwan University, Taipei 106, Taiwan \\ 3) Research Center for Developmental Biology and Regenerative Medicine, National Taiwan University, Taipei 106, Taiwan
}

(Received 22 May 2014/Accepted 24 October 2014/Published online in J-STAGE 12 November 2014)

ABSTRACT. Mesenchymal stem cells (MSCs) hold great potential in cell therapy and have attracted increasing interests in a wide range of biomedical sciences. However, the scarcity of MSCs and the prolonged isolation procedure limited the clinical application. To address these 2 issues, we developed a method to isolate MSCs from bone biopsy tissues of euthanized canine body donors. Compared to the traditional method to isolate MSCs from aspirated bone marrow (BMSCs), the isolation procedure for MSCs from harvested epiphyseal cancellous bone (EMSCs) was less time-consuming. The isolated EMSCs had similar plastic-adherence, tri-lineage differentiation and consistent surface marker profiles compared to BMSCs. We harvested BMSCs and EMSCs from 24 euthanized cases from clinics and 42 euthanized donors from a local shelter. The successful rate for EMSC isolation is significantly higher compared to BMSC isolation, while the other properties of the isolated MSCs including the clonogenicity, proliferative potentials and molecular phenotypes were not discernibly different between the MSCs established by the two methods. In conclusion, we demonstrated a new procedure to harvest MSCs by bone biopsy at the epiphyseal region. This method is less time consuming and more reliable, and the resulting MSCs are comparable to those harvested by bone marrow aspiration. The combination of the two methods can greatly improve the efficiency to harvest MSCs.

KEY WORDS: bone marrow, epiphyseal-derived mesenchymal stem cell, femur, humerus

doi: 10.1292/jvms.14-0265; J. Vet. Med. Sci. 77(3): 273-280, 2015

The therapeutic potential of mesenchymal stem cells (MSCs) has attracted increasing interest in a wide range of biomedical sciences. In laboratory experiments, canine MSCs have therapeutic effects in bone defects [4, 12], spinal cord injury [13, 21], myocardial infarction [26], chronic osteoarthritis [8] and cutaneous wounds [17]. Other than the use in bone healing, it is currently believed that the therapeutic effects of MSCs predominantly come from the paracrine secretions that modulate the topical immune environment, provide the survival signals and augment the endogenous regenerative potential at the injury sites. Accordingly, the applications of MSCs in many of the injuries, such as spinal cord injury, have to be in a timely manner [25]. Moreover, although the exact doses remain uncharacterized, more than several million cells per transplantation site are commonly used in the aforementioned laboratory experiments. Unfortunately, due to the small number of MSCs [1], ex vivo expansion might be necessary to achieve applicable numbers of MSCs and in turn may delay the timing of application. Therefore, new methods that improve the efficiency to harvest MSCs would benefit the clinical applications of MSCs.

Canine MSCs were first isolated from aspirated bone marrow and usually are termed bone marrow-derived MSCs

*Correspondence to: Liu, I-H., Department of Animal Science and Technology, National Taiwan University, Taipei 106, Taiwan. e-mail: ihliu@ntu.edu.tw

(C)2015 The Japanese Society of Veterinary Science

This is an open-access article distributed under the terms of the Creative Commons Attribution Non-Commercial No Derivatives (by-nc-nd) License $<$ http://creativecommons.org/licenses/by-nc-nd/3.0/>.
(BMSCs) [14]. As MSCs were isolated from most of the adult organs of the mice [5], canine MSCs were also reported to be isolated from a wide variety of sources including adipose tissue, periodontal ligament, umbilical cord vein, umbilical cord blood, Wharton's jelly, amniotic membrane, amniotic fluid, yolk sac and liver [7, 16, 18, 23, 24, 29, 33-35]. Accordingly, we previously reported a new method to isolate mice MSCs from the cancellous bone near the epiphyseal plate, termed epiphysis-derived MSCs (EMSCs) [3]. Although fitted in the minimum criteria for MSCs, mice EMSCs showed slightly different properties than BMSCs including the absence of major histocompatibility complex (MHC) -II, better clonogenicity and higher proliferative and differentiating potentials [3]. It is of interest to ascertain whether canine EMSCs are a better population of MSCs than BMSCs.

In this report, we aimed to translate our previous finding that MSCs can be harvested from mice epiphysis to establish a process to harvest the canine MSCs by bone biopsy from epiphyseal cancellous bone and to compare these MSCs with those obtained by bone marrow aspiration. Combining the harvest of MSCs via bone marrow aspiration and this new approach, canine MSCs can be harvested from 2 distinct but proximal anatomical locations to increase the harvested MSCs in a single procedure and hence improve the efficiency to harvest canine MSCs.

\section{MATERIALS AND METHODS}

Animals: Canine MSCs were harvested from 24 patient donors from National Taiwan University Veterinary Hospital (NTUVH) and 42 body donors from Taipei City Animal 


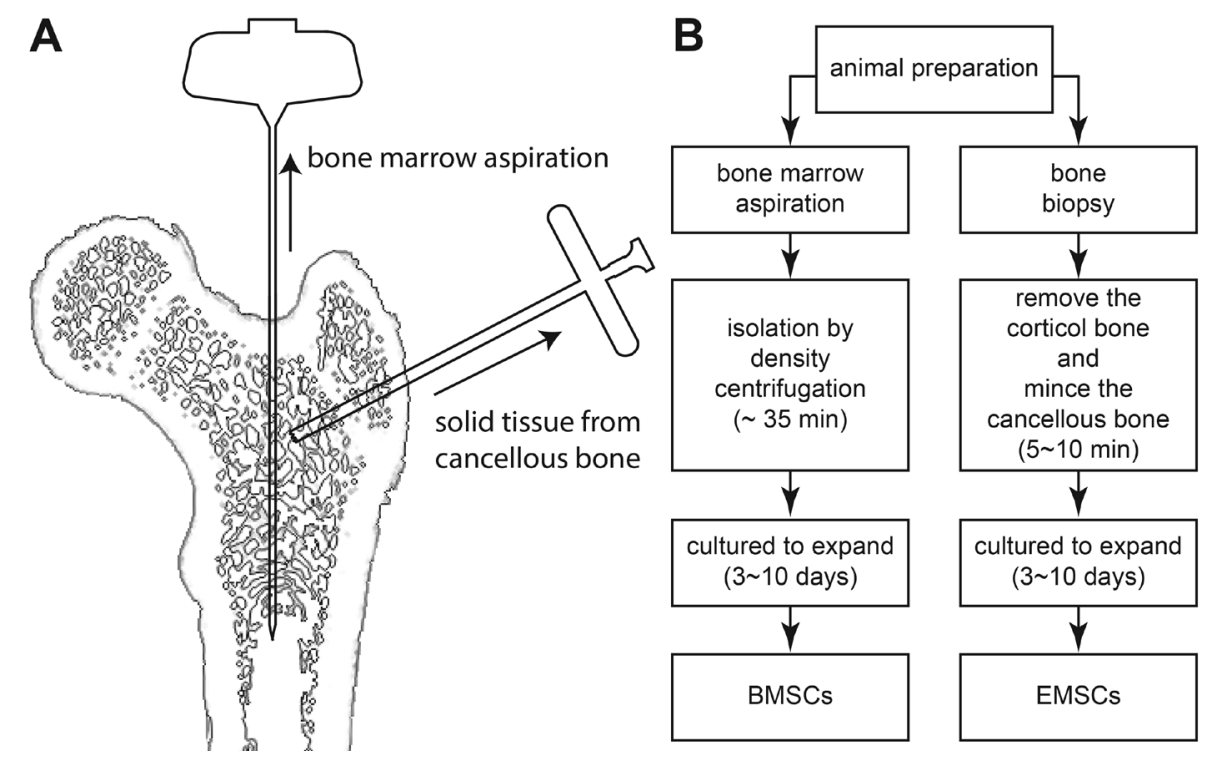

Fig. 1. Schematic comparisons of the harvest methods for BMSCs and EMSCs. (A) Approaching methods for bone marrow aspiration and bone biopsy for the isolation of BMSCs and EMSCs. (B) Flow diagram of the establishment of canine BMSCs and EMCSs.

Protection Office (TCAPO) and Taoyuan County Animal Disease Control Center (TCADCC). The patient donors were euthanized for various health conditions, and the owners of the patients agreed to participate in the pet body donation program at NTUVH. The donors from TCAPO and TCADCC were euthanized according to the Stray Dog Control Practices and Public Animal Shelter Regulations of Taiwan. The procedure reported in this work was reviewed and approved by the Institutional Animal Care and Use Committee, National Taiwan University (NTU-100-EL-1).

Isolation of canine MSCs by bone marrow aspiration and bone biopsy: The methods for isolation of canine MSCs described here were developed according to the previous methods used in mice and swine [3, 11]. Briefly, the skin over the proximal end of femur was shaved, sterilized and incised to expose the femur. Bone marrow biopsy needles (T-Lok $11 \mathrm{G} \times 4$ " Bone Marrow Biopsy Needle, Angiotech, Vancouver, Canada) were used to aspirate the bone marrow into $20 \mathrm{~m} l$ syringes containing 1,500 I.U. heparin (B. Braun Melsungen AG, Melsungen, Germany) (Fig. 1A). A trephine was used to perform bone biopsy near the suspected region of the growth plate at the proximal end of the femur (Fig. 1A). The harvested bone tissues were then transferred into culture medium once harvested. The bone marrow and biopsied bone tissues were kept on ice until transport to the laboratory for further isolation.

To isolate BMSCs (Fig. 1B), the aspirated bone marrow was diluted with 3 times its volume with phosphate buffered saline (PBS; 0780, Amresco, OH, U.S.A.) containing 2\% fetal bovine serum (FBS; SH30070, Hyclone, UT, U.S.A.), and the mononuclear cells were separated by cell density gradient centrifugation (Ficoll-paque; GE Healthcare, Uppsala, Sweden). The centrifugation was started at $210 \times g$ and increased by
$25 \times g$ every $30 \mathrm{sec}$ up to $510 \times g$ for $20 \mathrm{~min}$. The mononuclear cells were then transferred to $10 \mathrm{ml}$ of complete culture medium and centrifuged at $300 \times g$ for $8 \mathrm{~min}$ for washing. The resulting cell pallet was resuspended and cultured in one 10-cm plastic culture dish (TPP, Trasadingen, Switzerland) for every $2 \mathrm{~m} l$ bone marrow aliquot. The complete culture medium consisted of alpha-MEM (M0894, Sigma-Aldrich, St. Louis, MO, U.S.A.) supplemented with 16.7\% FBS, $3.7 \mathrm{mg} /$ $\mathrm{m} l$ sodium bicarbonate $\left(\mathrm{NaHCO}_{3} ; \mathrm{S} 5761\right.$, Sigma-Aldrich), $100 \mathrm{U} / \mathrm{m} l$ penicillin and $100 \mu \mathrm{g} / \mathrm{m} l$ streptomycin (Invitrogen, Carlsbad, CA, U.S.A.) and incubated in a humidified atmosphere containing $95 \%$ air and $5 \% \mathrm{CO}_{2}$ at $37^{\circ} \mathrm{C}$.

To isolate EMSCs (Fig. 1B), the cortical bone was removed from the bone biopsy after washed in PBS containing $2 \% \mathrm{FBS}$, and the spongy bone tissues were minced by scissors while washed twice with complete culture medium before cultured in a $10-\mathrm{cm}$ plastic culture dish in complete culture medium. The non-adherent cells in both preparations of BMSCs and EMSCs were removed by changing the medium every 3 days. Both preparations of MSCs were expanded for at least 2 passages and cryopreserved in FBS containing 10\% dimethyl sulfoxide (DMSO; D2650, Sigma-Aldrich).

Tri-lineage differentiation assay: To evaluate the adipogenic differentiation potentials, the MSCs were cultured to 50\% confluence and cultured in adipogenic induction medium consisting of alpha-MEM supplemented with 10\% FBS, $10 \mu \mathrm{g} /$ $\mathrm{m} l$ insulin (I6634, Sigma-Aldrich) $1 \mu \mathrm{M}$ dexamethasone (D4902, Sigma-Aldrich), $0.5 \mathrm{mM}$ isobutyl-methylxanthine (IBMX; I5879, Sigma-Aldrich) and $100 \mu \mathrm{M}$ indomethacin (I7378, Sigma-Aldrich) for 30 days with the induction medium changed every 3 days. After 30 days, cells were fixed with 10\% formalin (H121-08, Macron chemicals, Center Valley, PA, U.S.A.) for $10 \mathrm{~min}$, and lipid droplets were stained 
Table 1. Primers used in the RT-PCR

\begin{tabular}{|c|c|c|}
\hline Target gene & Genbank accession no. & Sequence \\
\hline \multirow[t]{2}{*}{$\beta$-actin ${ }^{\mathrm{a}}$} & NM_001195845 & Forward: GTCCACCTTCCAGCAAATGT \\
\hline & & Reverse: AAAGCCATGCCAATCTCATC \\
\hline \multirow[t]{2}{*}{$\mathrm{CD} 73^{\mathrm{a}}$} & XM_532221 & Forward: TGATCTTTCCCGAAAACCTG \\
\hline & & Reverse: TGGAATCCATCTCCACCATT \\
\hline \multirow[t]{2}{*}{$\mathrm{CD} 105^{\mathrm{a}}$} & XM_846376 & Forward: CCAATGCTACCGTGGAAGTT \\
\hline & & Reverse: GTTCCGTGGTGTTGACTCCT \\
\hline \multirow[t]{2}{*}{ MHC-1 [28] } & DQ469801 & Forward: CTCCCACTCCCTGAGGTATT \\
\hline & & Reverse: CGTCGTCTCCAGGTAGTTCC \\
\hline \multirow[t]{2}{*}{ MHC-2 [28] } & NM_001011723 & Forward: TGACTGTGCTCTCAAACACCC \\
\hline & & Reverse: TAATGATGCCCACCAGACCC \\
\hline
\end{tabular}

a) These primers were designed using Primer3 [31].

by Oil Red O (ORO; O9755, Sigma-Aldrich) staining [2, 3].

To evaluate the osteogenic differentiation potentials, MSCs were cultured to $50 \%$ confluence and then cultured in osteogenic induction medium consisting of alpha-MEM supplemented with $10 \% \mathrm{FBS}, 0.1 \mu \mathrm{M}$ dexamethasone, $10 \mathrm{mM}$ glycerol-2-phosphate (G9891, Sigma-Aldrich) and $50 \mu \mathrm{M}$ ascorbate-2-phosphate (A8960, Sigma-Aldrich) for 7 days. The induction medium was changed every 3 days, and the bone matrix mineralization was evaluated by Alizarin red $\mathrm{S}$ (ARS; A5533, Sigma-Aldrich) staining after fixation with $10 \%$ formalin [3].

To evaluate the chondrogenic differentiation potentials, $2 \times 10^{5}$ of MSCs were seeded in $15 \mathrm{ml}$ tubes. After centrifugation at $300 \mathrm{~g}$ for $5 \mathrm{~min}$, the cells were cultured in chondrogenic induction medium consisting of alpha-MEM supplemented with $1 \%$ FBS, $10 n \mathrm{~g} / \mathrm{m} l$ TGF- $\beta 1$ (240B, R\&D Systems, Minneapolis, MN, U.S.A.), $6.25 \mu \mathrm{g} / \mathrm{m} l$ insulin and $50 \mathrm{nM}$ ascorbate-2-phosphate for 21 days. The cell mass was fixed with $10 \%$ formalin and prepared for paraffin histological tissue sections and evaluated by Toluidine Blue O staining (TBO; T3260, Sigma-Aldrich) [3].

Surface marker profile analysis: Cells were incubated with FITC- or PE-conjugated monoclonal antibodies against CD73, CD34 (12-0340, eBioscience, San Diego, CA, U.S.A.), CD45R (12-5451, eBioscience), CD44 (11-5440, eBioscience) and CD90 (12-5900, eBiosciences) for 30 min at $4{ }^{\circ} \mathrm{C}$ in the dark. After washing with PBS containing $2 \%$ FBS and fixation with PBS containing 2\% FBS and $1 \%$ paraformaldehyde, ten thousand events were acquired using a flow cytometer (Beckman Coulter FC500) and analyzed by FCS Express software (Version 4.0; Denovo software). All experiments included negative controls that stained without antibodies and with isotype controls (mouse IgG1 K Isotype Control FITC, 11-4714; mouse IgG1 K Isotype control PE, 12-4714; eBioscience).

Colony formation efficiency assay: To evaluate the clonogenicity, the MSCs were plated at a density of 200 cells/ well in 6-well culture dishes. After 10 days of incubation, the colonies formed were fixed by methanol (32213, SigmaAldrich) and stained with Giemsa solution (10092-013, Invitrogen) [27]. A colony with a diameter more than $2 \mathrm{~mm}$ was defined as a colony-forming unit (CFU), and CFUs were enumerated under a light microscope.
Population doubling time (PDT): To evaluate the proliferative potential, the growth curve and cell numbers of MSCs harvested from NTUVH (\#17) and TCAPO (\#1, $\# 2$, \#16 and \#18) were determined by MTT assay. Briefly, MSCs (1,000 cells/well) were seeded into 96-well plates and grew for up to 6 days. Cell numbers were determined using the 3-(4,5-dimethyl-2-thiazolyl)-2,5-diphenyl-2Htetrazoliumbromide (MTT) assay (M5655, Sigma-Aldrich) following manufacturer's instruction. Each sample included five replications at every time point. The PDT was calculated according to the equation: $\mathrm{PDT}=\mathrm{t} /[(\log \mathrm{Nt}-\log \mathrm{No}) / \log 2]$, where $\mathrm{Nt}$ is the number of cells harvested, No is the number of cells inoculated, and $t$ is the time of the culture in hours [3].

$R T-P C R$ : To evaluate the expression of specific mRNA, total cellular RNA was extracted using the TRIzol reagent (15596-018, Invitrogen) and then treated with RNase free DNase (M610, Promega, Madison, MI, U.S.A.) according to manufacturer's instructions. Reverse transcription reactions were performed with $2 \mu \mathrm{g}$ total RNA using the SuperScript First-Strand Synthesis System (18080-044, Invitrogen), according to the manufacturer's instructions. PCR was performed with $1 \mu l$ of the single-stranded cDNA sample with $0.25 \mu l$ of Taq DNA polymerase (11615-010, Invitrogen), $1 \mu l$ of dNTP mix, $2 \mu l$ of forward and reversed primers respectively, $5 \mu l$ of polymerase reaction buffer, $1.5 \mu l$ of $50 \mathrm{mM} \mathrm{MgCl} 2$ and $37.25 \mu \mathrm{l}$ of water. The PCR program started at $94^{\circ} \mathrm{C}$ for $2 \mathrm{~min}$ followed by 30 cycles of $94^{\circ} \mathrm{C}$ for $30 \mathrm{sec}, 60^{\circ} \mathrm{C}$ for $1 \mathrm{sec}$ and $72^{\circ} \mathrm{C}$ for $40 \mathrm{sec}$ and ended with $72^{\circ} \mathrm{C}$ for $7 \mathrm{~min}$. The resulting amplicons were examined by agrarose gel electrophoresis. The sequences of the primers used in this study are as listed in Table 1.

Statistical analysis: To compare the isolation success rates of BMSCs and EMSCs from each donor, Wilcoxon Matched-pairs signed-ranks test was used to detect the statistical significance. To assess the effects of age and gender on the success rates, chi-squared test was performed. To evaluate whether biopsy weight and donor body weight affect the success rates of EMSCs harvest, the subjects were separated into three ranking groups according to the weight and Spearman's rank correlation coefficient was calculated for nonparametric dependency analysis. For all other comparisons, Mann-Whitney U test was used. In all statistical analyses, a $P$-value less than 0.05 was considered statisti- 

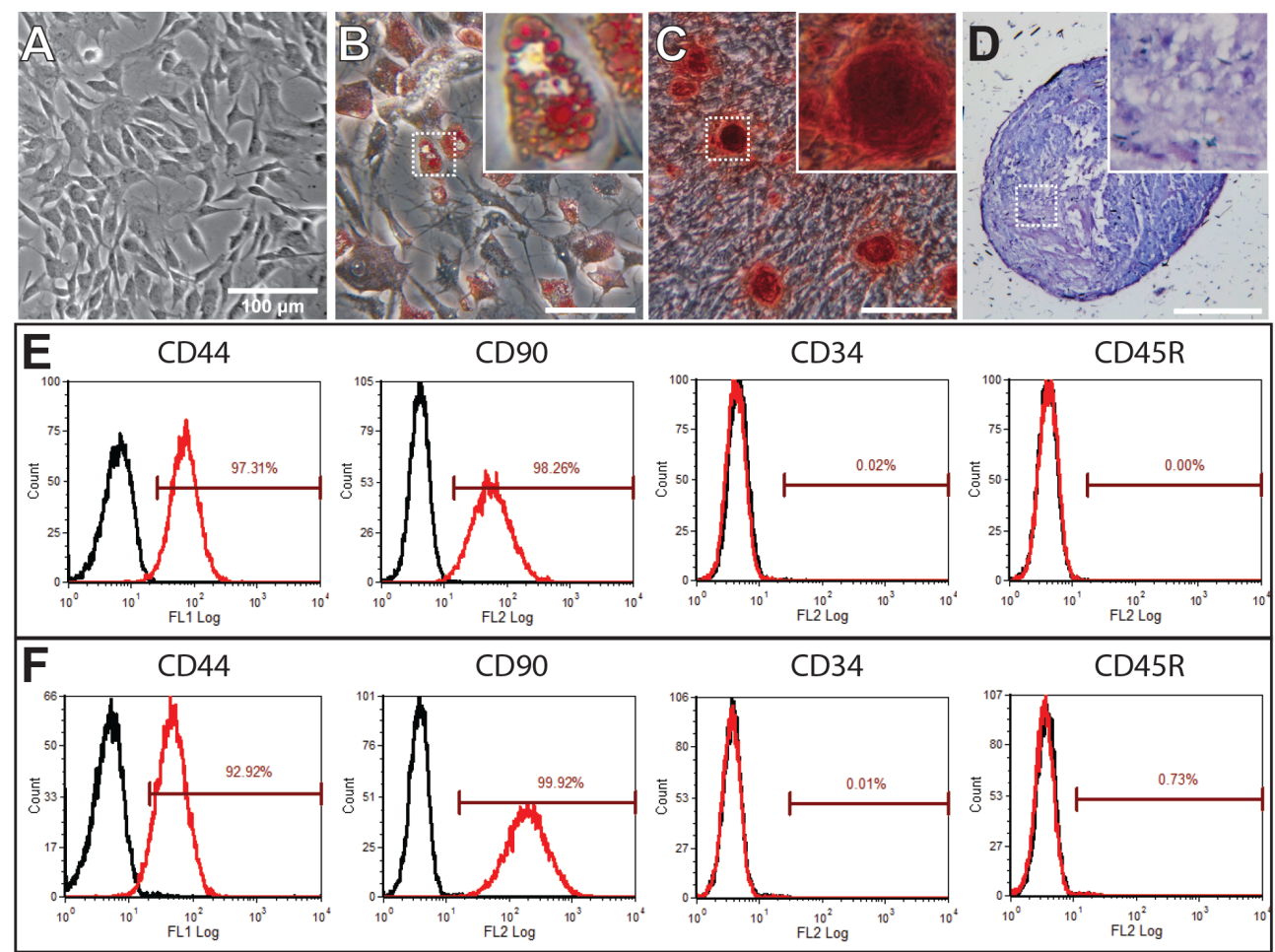

Fig. 2. Characterization of canine EMSCs. According to the minimum criteria for MSCs, EMSCs were plasticadherent with spindle-shape (A), were able to be induced for adipogenic (B), osteogenic (C) and chondrogenic (D) differentiation and showed specific profiles of surface markers (E). Insets: higher magnification of adipogenic (B), osteogenic (C) and chondrogenic (D) differentiations enclosed by dotted squares. The surface marker profiles of EMSCs were identical to those of BMSCs (F). Scale bars (A-D) are $100 \mu \mathrm{m}$.

cally significant, and the average values were presented as mean \pm standard error of the mean.

\section{RESULTS}

To establish a procedure that harvests both EMSCs and BMSCs from one cadaver, we adopted the isolation protocols for porcine BMSCs [11] and mice EMSCs [3]. In human clinical practice, bone marrow aspiration is usually performed at the iliac crest due to the ease of operation and the conversion of red marrow (medulla ossium rubra) into yellow marrow (medulla ossium flava) in proximal humerus and femur at older ages [22]. In canine veterinary clinical practice, bone marrow aspiration is suggested in all three locations [32]. However, the iliac crest is narrow and can be difficult for bone marrow aspiration in small dogs. Our team performed bone marrow aspiration at the proximal femur in order to maintain the consistency of the procedures among different dog breeds (Fig. 1).

Although we do not know the exact physiological location of EMSCs in the proximal femur, our previous work on mice and our experience on dogs showed that EMSCs reside at the cancellous bone at epiphysis near the epiphyseal plate (Fig. 1A). BMSCs are commonly isolated by density centrifugation in which the BMSCs are among the mononuclear cells in one of the gradient layers. This procedure usually takes about $30 \mathrm{~min}$, and multiple rounds of centrifugation sometimes is performed if higher purity of the mononuclear cells are interested (Fig. 1B). To isolate EMSCs, the removal of cortical bone from the bone biopy sample and the mince of the cancellous bone are the only operations needed before seeding to the culture dish. This procedure takes less than 5 min for each sample (Fig. 1B). The EMSCs spontaneously migrate out of the minced cancellous bones, adhere to the plastic surface of the culture dish and expand their numbers during the following culture period.

In accordance with the minimal criteria of definition for MSCs [6], EMSCs were consistently demonstrated to be adherent to the plastic surface with spindle-like shape (Fig. 2A) and differentiated into 3 lineages, adipocytes (Fig. 2B), osteoblasts (Fig. 2C) and chondroblasts (Fig. 2D). We obtained and tested 5 antibodies for surface markers that were commonly used to examine MSCs in mice and humans. Among the positive markers in human and mice BMSCs, CD73 did not provide consistent results using different lots of antibodies in the same cells (data not shown). Two negative markers (CD34 and CD45R) and two positive markers (CD44 and CD90) were included as our standard surface marker profile for EMSCs (Fig. 2E) and BMSCs (Fig. 2F).

In this report, the procedure of initial isolation and expan- 
Table 2. Patient body donors

\begin{tabular}{|c|c|c|c|c|c|c|c|c|}
\hline Number & Age & Breed & Gender & Health condition & BMSCs & EMSCs & $\begin{array}{l}\text { Bone } \\
\text { biopsy } \\
\text { (g) }\end{array}$ & $\begin{array}{c}\text { Body } \\
\text { weight } \\
(\mathrm{kg})\end{array}$ \\
\hline 1 & 2 & Husky & M & spinal fracture and subluxation due to accident & + & + & N/A & N/A \\
\hline 2 & 2 & Yorkshire & $\mathrm{F}$ & CNS inflammatory disease (NME)* & - & + & N/A & 1 \\
\hline 3 & 6 & Beagle & M & bladder and testicle tumor & + & + & $\mathrm{N} / \mathrm{A}$ & 13.8 \\
\hline 4 & 6 & Shiba Inu & M & $\mathrm{N} / \mathrm{A}$ & - & + & $\mathrm{N} / \mathrm{A}$ & 1.9 \\
\hline 5 & N/A & N/A & $\mathrm{F}$ & spinal fracture and subluxation due to accident & - & + & N/A & N/A \\
\hline 6 & 4 & Dachshund & M & spinal subluxation due to accident & + & + & $\mathrm{N} / \mathrm{A}$ & 4.3 \\
\hline 7 & 13 & Mixed & $\mathrm{F}$ & brain disease & + & + & N/A & N/A \\
\hline 8 & 7 & Gold Retriever & M & suspected spinal meningioma* & - & + & N/A & 31.5 \\
\hline 9 & 9 & Lab Retriever & M & cryptorchidism, testical tumor & - & $x$ & N/A & 30.8 \\
\hline 10 & 10 & Mixed & $\mathrm{F}$ & suspected meningioma & + & + & N/A & 30 \\
\hline 11 & 10 & N/A & M & brain tumor & - & $x$ & N/A & 16.2 \\
\hline 12 & $8 \mathrm{~m} / \mathrm{o}$ & N/A & M & spinal fracture due to accident & + & + & N/A & N/A \\
\hline 13 & 10 & Beagle & N/A & tumor metastasis & + & + & N/A & 12.25 \\
\hline 14 & 7 & Maltese & $\mathrm{F}$ & uroabdomen, vulvar tumor & - & + & 0.18 & N/A \\
\hline 15 & 9 & Golden Retriever & M & severe brain disease of unknown cause & - & + & 0.55 & 32 \\
\hline 16 & 16 & Shih Tzu & $\mathrm{F}$ & abdominal tumor & - & + & 0.46 & N/A \\
\hline 17 & 8 & Mix & $\mathrm{F}$ & renal transplant for experiments, healthy dog & + & + & 0.8 & N/A \\
\hline 18 & 3 & Schnauzer & M & canine distemper & + & + & 0.4 & 6.02 \\
\hline 19 & $3 \mathrm{~m} / \mathrm{o}$ & Mix & M & canine distemper & - & + & 0.12 & 2.72 \\
\hline 20 & 2 & Poodle & $\mathrm{F}$ & canine distemper & - & + & 0.34 & 4.34 \\
\hline 21 & 8 & Golden Retriever & $\mathrm{F}$ & $\mathrm{C} 4 / \mathrm{C} 5$ intervertebral disc disease & $!$ & + & 0.34 & 47.1 \\
\hline 22 & 8 & Mix & M & nasal tumor suspected & + & + & 0.5 & 18 \\
\hline 23 & $3 \mathrm{~m} / \mathrm{o}$ & Mix & M & canine distemper & + & $!$ & 0.22 & 4.52 \\
\hline 24 & 8 & Mix & M & right back mass of unknown origin, hindlimb paralysis & + & + & 0.3 & 38 \\
\hline
\end{tabular}

+: MSCs harvest successful; -: Fail to obtain bone marrow; !: MSCs fail to expand; *: Long-term steroids; $\times$ : Microorganism contamination; N/A: Information not available.

sion before any trypsinization was defined as passage 0 (P0), which took at most 10 days. A successful establishment of passage 1 (P1), i.e., MSCs that showed no sign of contamination, was defined as a successful isolation. Among 24 patient donors from NTUVH (Fig. 3A) (Table 2) and 42 body donors from stray animal shelter of TCAPO and TCADCC (Fig. 3B) (Table 3), we recorded a significantly lower successful rate for the isolation of BMSCs compared to EMSC isolation $(P=0.0117$ at NTUVH; $P=0.0352$ at stray animal shelter). Failures in harvesting BMSCs were predominantly due to the failure of bone marrow aspiration $(65.6 \%$ among all failure cases). In contrast, failures in harvesting EMSCs were predominantly because of the contamination by microorganisms (64.3\% among all failure cases). The other reason accounted for the failure is the failure of obtained samples to grow into $\mathrm{P} 0$ culture ( $>10 \%$ confluency) within 10 days.

Age (less than 4-year-old vs. more than 7-year-old; $P=0.7824$ for BMSCs; $P=0.0885$ for EMSCs) and gender (male vs. female; $P=0.2646$ for BMSCs; $P=0.1364$ for EMSCs) were not found to significantly affect the harvest of BMSCs and EMSCs. Interestingly, the failure of bone marrow aspiration in NTUVH $(45.83 \%$ among all cases, $n=24$ ) was significantly higher than in stray animal shelters ( $13.51 \%$ among all cases, $n=37$ excluding not attempted, $P=0.0075$ ), implying that health conditions might be partially responsible for the failure of bone marrow aspiration. It is reasonable to speculate that body weight might affect the
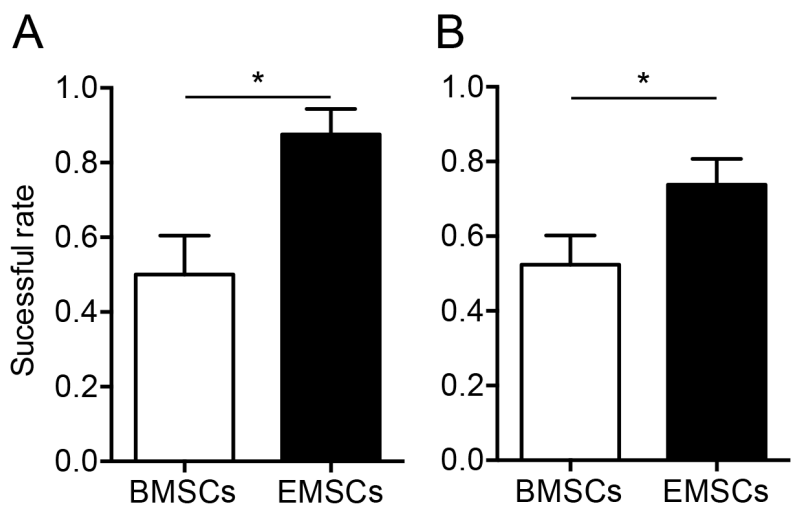

Fig. 3. Successful rates in establishing canine EMSCs and BMSCs. The successful rate was significantly lower in the isolation of BMSCs compared to EMSCs at both NTUVH (A) and stray animal shelters (B). ${ }^{*} P<0.05$

size of bone biopsy and the bigger size of bone biopsy might contribute to higher success rate of EMSCs harvest. However, we found that both body weight (Spearman's Rho $=0.5$, $P>0.999$ for BMSCs; Spearman's Rho $=-0.5, P>0.999$ for EMSCs) and the net weight of bone biopsy (Spearman's Rho $=1.0, P=0.333$ ) did not significantly correlate with the success rate of EMSCs harvest. 
Table 3. Donors from animal shelter

\begin{tabular}{|c|c|c|c|c|}
\hline Number & Estimated age (y/o) & BMSCs & EMSCs & Bone biopsy $(\mathrm{g})$ \\
\hline 1 & $>8$ & + & + & $\mathrm{N} / \mathrm{A}$ \\
\hline 2 & $<3$ & + & + & N/A \\
\hline 3 & $>8$ & + & + & N/A \\
\hline 4 & $>8$ & $!$ & $x$ & N/A \\
\hline 5 & $<3$ & + & + & N/A \\
\hline 6 & $<3$ & + & $x$ & N/A \\
\hline 7 & $>8$ & + & + & $\mathrm{N} / \mathrm{A}$ \\
\hline 8 & $<3$ & + & + & N/A \\
\hline 9 & $<3$ & + & + & N/A \\
\hline 10 & $>8$ & - & + & N/A \\
\hline 11 & $>8$ & + & + & N/A \\
\hline 12 & $<3$ & + & + & N/A \\
\hline 13 & $<3$ & + & + & N/A \\
\hline 14 & $<3$ & + & + & N/A \\
\hline 15 & $>8$ & $x$ & + & N/A \\
\hline 16 & $>8$ & + & + & N/A \\
\hline 17 & $>8$ & - & + & N/A \\
\hline 18 & $>8$ & + & + & N/A \\
\hline 19 & $>8$ & + & + & N/A \\
\hline 20 & $2 \mathrm{~m} / \mathrm{o}$ & $!$ & $!$ & 0.26 \\
\hline 21 & $4 \mathrm{~m} / \mathrm{o}$ & $!$ & + & 0.28 \\
\hline 22 & $8-9$ & + & + & 0.48 \\
\hline 23 & $7-8$ & + & + & 0.4 \\
\hline 24 & $5-6$ & + & + & 0.38 \\
\hline 25 & $5-6$ & $!$ & + & 0.29 \\
\hline 26 & $2 \mathrm{~m} / \mathrm{o}$ & - & $x$ & 0.28 \\
\hline 27 & $2 \mathrm{~m} / \mathrm{o}$ & - & $x$ & 0.35 \\
\hline 28 & $7-8$ & $\triangle$ & + & 0.64 \\
\hline 29 & $6 \mathrm{~m} / \mathrm{o}$ & - & $x$ & 0.2 \\
\hline 30 & $1-3$ & - & ! & 0.32 \\
\hline 31 & $6 \mathrm{~m} / \mathrm{o}$ & $\triangle$ & + & 0.3 \\
\hline 32 & $7-8$ & $!$ & $\times$ & 0.5 \\
\hline 33 & $3 \mathrm{~m} / \mathrm{o}$ & + & $\times$ & 0.25 \\
\hline 34 & $1-3$ & $!$ & $!$ & 0.34 \\
\hline 35 & $1-3$ & + & $!$ & 0.3 \\
\hline 36 & $1-3$ & + & + & 0.15 \\
\hline 37 & $1-3$ & $!$ & + & 0.2 \\
\hline 38 & $7-8$ & $!$ & + & 0.4 \\
\hline 39 & $7-8$ & $!$ & + & 0.46 \\
\hline 40 & $7-8$ & + & + & 0.4 \\
\hline 41 & $6 \mathrm{~m} / \mathrm{o}$ & $\triangle$ & + & 0.3 \\
\hline 42 & $6 \mathrm{~m} / \mathrm{o}$ & $\triangle$ & + & 0.4 \\
\hline
\end{tabular}

+: MSCs harvest successful; !: MSCs fail to expand; -: Fail to obtain bone marrow; $\times$ : Microorganism contamination; $\triangle$ : Bone marrow aspiration not attempted; N/A: Information not available.

Our previous report on mice suggested that the MSCs established by the two methods represent cells with different properties and that EMSCs have greater density of clonogenicity with higher proliferative potential [3]. Due to the anatomical proximity, it is of interest to clarify whether the populations of the canine MSCs harvested by bone marrow aspiration and bone biopsy are distinct. Surprisingly, we did not find a significant difference in the colony forming efficiency (CFE) between the MSCs harvested by the two methods (Fig. 4A, Supplementary Table 1). In addition, the
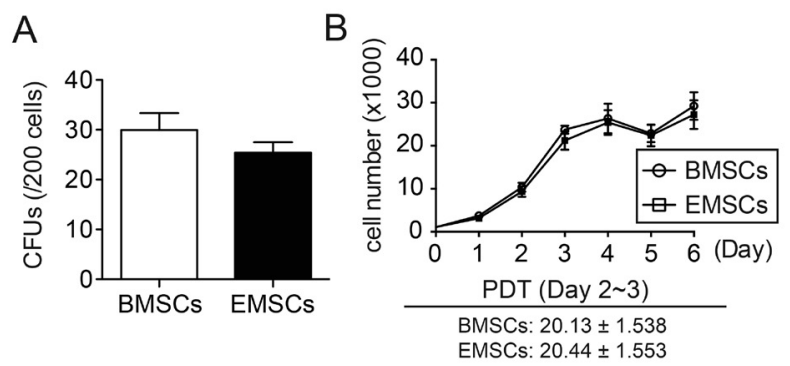

C

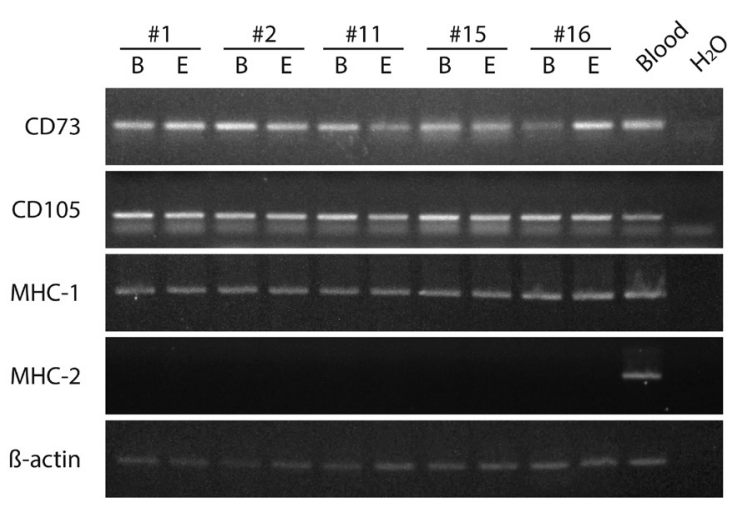

Fig. 4. Comparisons between EMSCs and BMSCs. (A, B) The MSCs established by bone biopsy and bone marrow aspiration had similar CFE (A) and PDT (B). (C) RT-PCR indicated that both BMSCs (B) and EMSCs (E) from donor \#1, \#2, \#11, \#15 and \#16 at TCAPO express CD73 and CD105, which are typical MSCs positive markers. Moreover, all MSCs express MHC-1, but not MHC-2. There was no discernible difference between the MSC populations established by these two methods. $\beta$-actin was used as the loading control. A blood sample from donor \#27 (Blood) at TCAPO was used as a positive control, while no template reactions $\left(\mathrm{H}_{2} \mathrm{O}\right)$ were done as a negative control to all reactions markers.

population doubling time (PDT) was also similar between the two groups (Fig. 4B).

In the mouse, one of the major differences between EMSCs and BMSCs is the lack of MHC-2 in EMSCs. To ascertain the difference between BMSCs and EMCSs, reverse transcription-polymerase chain reaction (RT-PCR) was performed in 5 randomly selected donors from TCAPO that provided both BMSCs and EMSCs. All BMSCs and EMSCs expressed CD73 and CD105, which are commonly used as positive markers for MSCs (Fig. 4C). Furthermore, all samples were positive for MHC-1, while negative for MHC-2 (Fig. 4C). These expression profiles are identical to a typical mouse and human BMSCs $[3,20]$, and there is no discernible difference between the canine MSCs established via bone marrow aspiration and bone biopsy.

\section{DISCUSSION}

In this report, we attempted to translate our previous findings in mice that MSCs can be obtained from cancellous bones into canine applications. According to our observation, shearing the entire femoral head did not effectively 
increase the harvested MSCs compared to multiple biopsies taken near the epiphyseal plate. We speculated that the MSCs in the cancellous bone were restricted to a subregion near the epiphyseal plate. This method is also suitable for the clinical practice, if autologous MSCs are to be obtained. Furthermore, this method can easily be performed in combination with traditional bone marrow aspiration. While establishing MSCs from bone marrow aspiration (BMSCs) requires gradient centrifugation and medium changes, the harvest of EMSCs requires only the harvested tissue to be minced, which saves time and effort during the isolation procedure. However, since bone biopsy creates a lesion near the epiphyseal plate, the weakened bone structure might result in fracture and could severely compromise the life quality of the patients. This could be a limitation on autologous EMSCs.

It has been recognized that MSCs are a heterogeneous cell population, which is why minimal criteria is required to define this cell type. Among the minimal criteria, specific surface markers have been a widely used method in MSCs in mice and humans. Unfortunately, the specific surface marker profile is not consistent across various animal species. Divergent expression profiles of surface markers have been reported in canine MSCs using several different experimental techniques $[9,15,30]$. Only negative markers, such as CD34 and CD45, are consistently negative throughout every publication. Some markers, such as CD29 and STRO-1, are positive in one study, but these results require more examination $[9,30]$. CD90 has been a controversial positive marker in studies of canine BMSCs [9, 15, 30], and our result indicated a high percentage of positive gated cells. All these variations could be due to the heterogeneous idiosyncrasy of canine immunology among various breeds, the methodologies of isolation or ex vivo culture conditions.

In the veterinary hospital, most euthanasia of patients was due to severe health conditions with poor quality of life, and some of these dogs had been on long-term medications that might potentially contribute to the lower successful isolation rate of BMSCs from donors at NTUVH. Failure of the aspiration from bone marrow is one of the major signs of bone marrow fibrosis, which might be caused by age and other health disorders. Accordingly, our results showed significantly higher failure rate of bone marrow aspiration in NTUVH than in stray animal shelters, implying that health conditions might be partially responsible for the failure of bone marrow aspiration. Other than the health conditions, the delay of bone marrow aspiration after euthanasia might be the other reason. We noticed that the pet owners often accompanied the body donors for the moment of grief after euthanasia and this may result in extreme hypoxia, formation of blood clots and, in turn, the failure of the aspiration of bone marrow. Due to the lack of precise records and study design to draw a conclusion, we observed that it was more difficult to successfully perform bone marrow aspiration after 90 min post-mortem and this was why we omitted few bone marrow aspirations in stray animal shelter when the harvest procedure was delayed more than 90 min after euthanasia (Table 3). In comparison, our results showed that all the complexities causing the failure of BMSC harvest had no influence on EMSC harvest, indicat- ing that EMSC harvest provides a more reliable approach to isolate canine MSCs in the clinics.

Our results indicated that there is no discernible difference between BMSCs and EMSCs with both MSCs expressing MHC-1 but not MHC-2. Interestingly, a recent study suggested that the incompatibility of MHC does not significantly increase the rejection risk of transplanted MSCs in dogs: $50 \%$ of recipient dogs tolerated the MSCs with unrelated MHC [19]. Recent model in explaining the rejection in allogeneic transplantation suggested the presence of MHC-1 but not MHC-2 provided an NK cell inhibitory signal [10]. This might explain the immune tolerance of MSCs when transplanted into the recipients allogeneically.

Taken together, we reported a method to harvest canine MSCs by bone biopsy of the cancellous bone near the epiphyseal plate. This new approach is easy to perform and more reliable to obtain MSCs. The MSCs harvested by bone marrow aspiration and bone biopsy were indistinguishable. Since the operation sites are proximate to each other, combining this new method with the traditional BMSCs could effectively increase the harvest efficiency of MSCs.

ACKNOWLEDGMENTS. The authors would like to thank Dr. Harry Mersmann for the corrections of this manuscript, Dr. Shinn-Chih Wu for the technical support on MSCs isolation and Dr. Chun-Chun Cheng, Ms. Kai-Han Lin, Ke-Hsuan Wei and Tzu-Yu Hsu for the assistance on some experiments. This work was funded by National Health Research Institutes, Taiwan (NHRI-EX102-10116EC) to IHL. The funding agencies have no role in the experimental design, data acquisition, analysis and interpretation of this work.

\section{REFERENCES}

1. Caplan, A. I. 2007. Adult mesenchymal stem cells for tissue engineering versus regenerative medicine. J. Cell. Physiol. 213: 341-347. [Medline] [CrossRef]

2. Chang, Y. J., Shih, D. T., Tseng, C. P., Hsieh, T. B., Lee, D. C. and Hwang, S. M. 2006. Disparate mesenchyme-lineage tendencies in mesenchymal stem cells from human bone marrow and umbilical cord blood. Stem Cells 24: 679-685. [Medline] [CrossRef]

3. Cheng, C. C., Lian, W. S., Hsiao, F. S., Liu, I. H., Lin, S. P., Lee, Y. H., Chang, C. C., Xiao, G. Y., Huang, H. Y., Cheng, C. F., Cheng, W. T. and Wu, S. C. 2012. Isolation and characterization of novel murine epiphysis derived mesenchymal stem cells. PLoS ONE 7: e36085. [Medline] [CrossRef]

4. Cui, L., Liu, B., Liu, G., Zhang, W., Cen, L., Sun, J., Yin, S., Liu, W. and Cao, Y. 2007. Repair of cranial bone defects with adipose derived stem cells and coral scaffold in a canine model. Biomaterials 28: 5477-5486. [Medline] [CrossRef]

5. da Silva Meirelles, L., Chagastelles, P. C. and Nardi, N. B. 2006. Mesenchymal stem cells reside in virtually all post-natal organs and tissues. J. Cell Sci. 119: 2204-2213. [Medline] [CrossRef]

6. Dominici, M., Le Blanc, K., Mueller, I., Slaper-Cortenbach, I., Marini, F., Krause, D., Deans, R., Keating, A., Prockop, D. and Horwitz, E. 2006. Minimal criteria for defining multipotent mesenchymal stromal cells. The International Society for Cellular Therapy position statement. Cytotherapy 8: 315-317. [Medline] [CrossRef]

7. Filioli Uranio, M., Valentini, L., Lange-Consiglio, A., Caira, M., Guaricci, A. C., L’Abbate, A., Catacchio, C. R., Ventura, M., 
Cremonesi, F. and Dell'Aquila, M. E. 2011. Isolation, proliferation, cytogenetic, and molecular characterization and in vitro differentiation potency of canine stem cells from foetal adnexa: a comparative study of amniotic fluid, amnion, and umbilical cord matrix. Mol. Reprod. Dev. 78: 361-373. [Medline] [CrossRef]

8. Guercio, A., Di Marco, P., Casella, S., Cannella, V., Russotto, L., Purpari, G., Di Bella, S. and Piccione, G. 2012. Production of canine mesenchymal stem cells from adipose tissue and their application in dogs with chronic osteoarthritis of the humeroradial joints. Cell Biol. Int. 36: 189-194. [Medline] [CrossRef]

9. Hodgkiss-Geere, H. M., Argyle, D. J., Corcoran, B. M., Whitelaw, B., Milne, E., Bennett, D. and Argyle, S. A. 2012. Characterization and differentiation potential of bone marrow derived canine mesenchymal stem cells. Vet. J. 194: 361-368. [Medline] [CrossRef]

10. Höglund, P. and Brodin, P. 2010. Current perspectives of natural killer cell education by MHC class I molecules. Nat. Rev. Immunol. 10: 724-734. [Medline] [CrossRef]

11. Hsiao, F. S., Lian, W. S., Lin, S. P., Lin, C. J., Lin, Y. S., Cheng, E. C., Liu, C. W., Cheng, C. C., Cheng, P. H., Ding, S. T., Lee, K. H., Kuo, T. F., Cheng, C. F., Cheng, W. T. and Wu, S. C. 2011. Toward an ideal animal model to trace donor cell fates after stem cell therapy: production of stably labeled multipotent mesenchymal stem cells from bone marrow of transgenic pigs harboring enhanced green fluorescence protein gene. J. Anim. Sci. 89: 3460-3472. [Medline] [CrossRef]

12. Jang, B. J., Byeon, Y. E., Lim, J. H., Ryu, H. H., Kim, W. H., Koyama, Y., Kikuchi, M., Kang, K. S. and Kweon, O. K. 2008. Implantation of canine umbilical cord blood-derived mesenchymal stem cells mixed with beta-tricalcium phosphate enhances osteogenesis in bone defect model dogs. J. Vet. Sci. 9: 387-393. [Medline] [CrossRef]

13. Jung, D. I., Ha, J., Kang, B. T., Kim, J. W., Quan, F. S., Lee, J. H., Woo, E. J. and Park, H. M. 2009. A comparison of autologous and allogenic bone marrow-derived mesenchymal stem cell transplantation in canine spinal cord injury. J. Neurol. Sci. 285: 67-77. [Medline] [CrossRef]

14. Kadiyala, S., Young, R. G., Thiede, M. A. and Bruder, S. P. 1997. Culture expanded canine mesenchymal stem cells possess osteochondrogenic potential in vivo and in vitro. Cell Transplant. 6: 125-134. [Medline] [CrossRef]

15. Kamishina, H., Deng, J., Oji, T., Cheeseman, J. A. and Clemmons, R. M. 2006. Expression of neural markers on bone marrow-derived canine mesenchymal stem cells. Am. J. Vet. Res. 67: 1921-1928. [Medline] [CrossRef]

16. Kang, B. J., Ryu, H. H., Park, S. S., Koyama, Y., Kikuchi, M., Woo, H. M., Kim, W. H. and Kweon, O. K. 2012. Comparing the osteogenic potential of canine mesenchymal stem cells derived from adipose tissues, bone marrow, umbilical cord blood, and Wharton's jelly for treating bone defects. J. Vet. Sci. 13: 299-310. [Medline] [CrossRef]

17. Kim, J. W., Lee, J. H., Lyoo, Y. S., Jung, D. I. and Park, H. M. 2013. The effects of topical mesenchymal stem cell transplantation in canine experimental cutaneous wounds. Vet. Dermatol. 24: 242- e 253. [Medline] [CrossRef]

18. Kisiel, A. H., McDuffee, L. A., Masaoud, E., Bailey, T. R., Esparza Gonzalez, B. P. and Nino-Fong, R. 2012. Isolation, characterization, and in vitro proliferation of canine mesenchymal stem cells derived from bone marrow, adipose tissue, muscle, and periosteum. Am. J. Vet. Res. 73: 1305-1317. [Medline] [CrossRef]

19. Kornblit, B., Leisenring, W. M., Santos, E. B., Storb, R. and Sandmaier, B. M. 2013. Safety of treatment with DLA-identical or unrelated mesenchymal stromal cells in DLA-identical canine bone marrow transplantation. Chimerism 4: 95-101. [Medline] [CrossRef]

20. Le Blanc, K., Tammik, C., Rosendahl, K., Zetterberg, E. and
Ringden, O. 2003. HLA expression and immunologic properties of differentiated and undifferentiated mesenchymal stem cells. Exp. Hematol. 31: 890-896. [Medline] [CrossRef]

21. Lee, J. H., Chang, H. S., Kang, E. H., Chung, D. J., Choi, C. B., Lee, J. H., Hwang, S. H., Han, H. and Kim, H. Y. 2009. Percutaneous transplantation of human umbilical cord blood-derived multipotent stem cells in a canine model of spinal cord injury. $J$. Neurosurg. Spine 11: 749-757. [Medline] [CrossRef]

22. Małkiewicz, A. and Dziedzic, M. 2012. Bone marrow reconversion - imaging of physiological changes in bone marrow. Pol. J. Radiol. 77: 45-50. [Medline]

23. Neupane, M., Chang, C. C., Kiupel, M. and Yuzbasiyan-Gurkan, V. 2008. Isolation and characterization of canine adipose-derived mesenchymal stem cells. Tissue Eng. Part A 14: 1007-1015. [Medline] [CrossRef]

24. Park, S. B., Seo, M. S., Kim, H. S. and Kang, K. S. 2012. Isolation and characterization of canine amniotic membrane-derived multipotent stem cells. PLoS ONE 7: e44693. [Medline] [CrossRef]

25. Park, S. S., Byeon, Y. E., Ryu, H. H., Kang, B. J., Kim, Y., Kim, W. H., Kang, K. S., Han, H. J. and Kweon, O. K. 2011. Comparison of canine umbilical cord blood-derived mesenchymal stem cell transplantation times: involvement of astrogliosis, inflammation, intracellular actin cytoskeleton pathways, and neurotrophin-3. Cell Transplant. 20: 1867-1880. [Medline] [CrossRef]

26. Perin, E. C., Silva, G. V., Assad, J. A., Vela, D., Buja, L. M., Sousa, A. L., Litovsky, S., Lin, J., Vaughn, W. K., Coulter, S., Fernandes, M. R. and Willerson, J. T. 2008. Comparison of intracoronary and transendocardial delivery of allogeneic mesenchymal cells in a canine model of acute myocardial infarction. J. Mol. Cell. Cardiol. 44: 486-495. [Medline] [CrossRef]

27. Sarma, N. J., Takeda, A. and Yaseen, N. R. 2010. Colony forming cell (CFC) assay for human hematopoietic cells. J. Vis. Exp. 2195 10.3791/2195. [Medline]

28. Schäfer-Somi, S., Beceriklisoy, H. B., Budik, S., Kanca, H., Aksoy, O. A., Polat, B., Cetin, Y., Ay, S. S. and Aslan, S. 2008. Expression of genes in the canine pre-implantation uterus and embryo: implications for an active role of the embryo before and during invasion. Reprod. Domest. Anim. 43: 656-663. [Medline] [CrossRef]

29. Seo, M. S., Park, S. B. and Kang, K. S. 2012. Isolation and characterization of canine Wharton's jelly-derived mesenchymal stem cells. Cell Transplant. 21: 1493-1502. [Medline] [CrossRef]

30. Takemitsu, H., Zhao, D., Yamamoto, I., Harada, Y., Michishita, M. and Arai, T. 2012. Comparison of bone marrow and adipose tissue-derived canine mesenchymal stem cells. BMC Vet. Res. 8: 150. [Medline] [CrossRef]

31. Untergasser, A., Cutcutache, I., Koressaar, T., Ye, J., Faircloth, B. C., Remm, M. and Rozen, S. G. 2012. Primer3-new capabilities and interfaces. Nucleic Acids Res. 40: e115. [Medline] [CrossRef]

32. Villiers, E. and Blackwood, L., British Small Animal Veterinary Association. 2005. BSAVA Manual of Canine and Feline Clinical Pathology, 2nd ed., British Small Animal Veterinary Association, Quedgeley, Gloucester.

33. Wang, W. J., Zhao, Y. M., Lin, B. C., Yang, J. and Ge, L. H. 2012. Identification of multipotent stem cells from adult dog periodontal ligament. Eur. J. Oral Sci. 120: 303-310. [Medline]

34. Wenceslau, C. V., Miglino, M. A., Martins, D. S., Ambrosio, C. E., Lizier, N. F., Pignatari, G. C. and Kerkis, I. 2011. Mesenchymal progenitor cells from canine fetal tissues: yolk sac, liver, and bone marrow. Tissue Eng. Part A 17: 2165-2176. [Medline] [CrossRef]

35. Zucconi, E., Vieira, N. M., Bueno, D. F., Secco, M., Jazedje, T., Ambrosio, C. E., Passos-Bueno, M. R., Miglino, M. A. and Zatz, M. 2010. Mesenchymal stem cells derived from canine umbilical cord vein-a novel source for cell therapy studies. Stem Cells Dev. 19: 395-402. [Medline] [CrossRef] 